\title{
Heavy flavour studies with the ATLAS detector
}

\author{
Ina Chalupková on behalf of the ATLAS Collaboration
}

Charles University in Prague

Institute of Particle and Nuclear Physics

Hadron Structure 2015, 30.6.2015
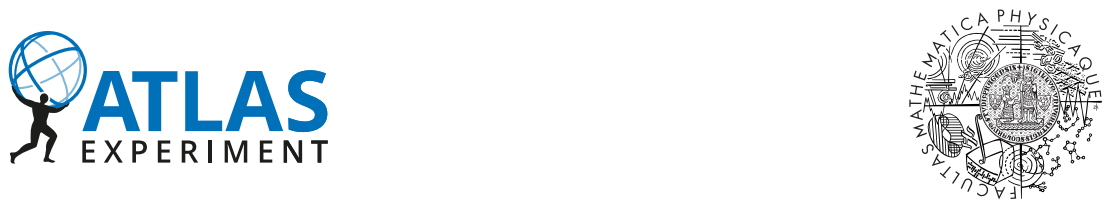


\section{ATLAS detector}

Inner Detector: tracking, momentum and vertex measurement

- $|\eta|<2.5, \mathrm{~d}_{0}$ resolution $\sim 10 \mu \mathrm{m}$

Muon Spectrometer: trigger and muon identification

- $|\eta|<2.7$

Mass resolution $\sigma\left(m_{\mathrm{J} / \psi}\right)=60 \pm 1 \mathrm{MeV}$
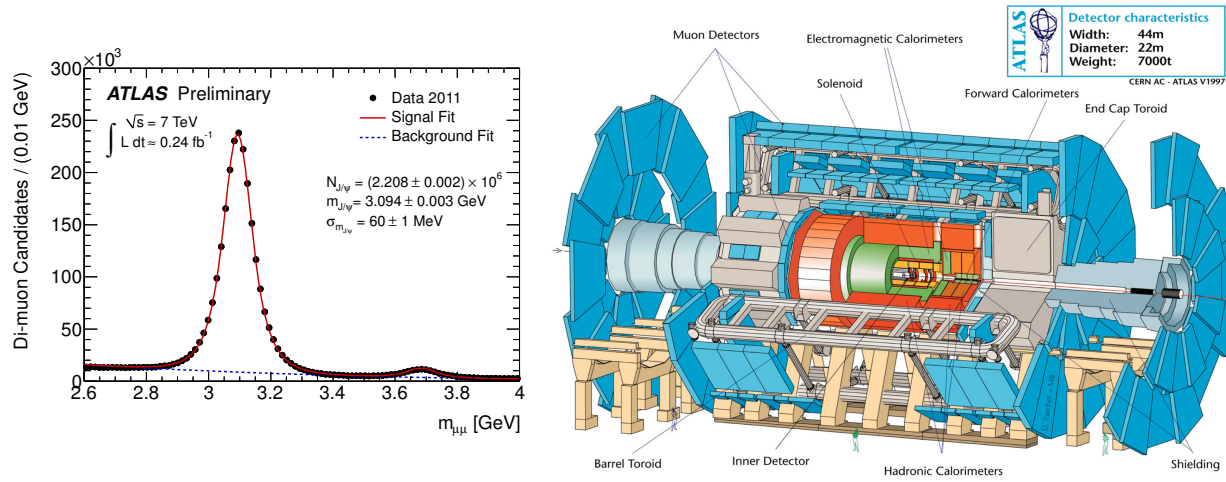


\section{Trigger and dataset}

B-physics trigger:

- muons, di-muon decays $(J / \psi, \Upsilon)$

- L1: single and di-muon triggers (threshold from $p_{\mathrm{T}}=4 \mathrm{GeV}$ )

- L2 and EF: muons from common vertex, opposite charge

- invariant mass window of $\mathrm{J} / \psi, B$ and $\Upsilon$ un-prescaled in 2011

- 2012: added barrel-only triggers to keep a larger fraction of data

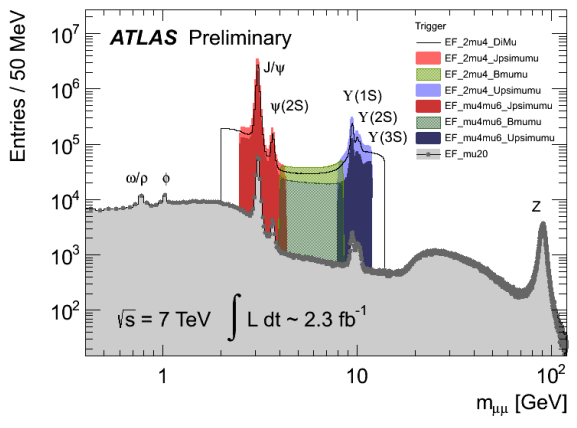

Datasets for analyses shown here:

- 2011 dataset at $\sqrt{s}=7 \mathrm{TeV}$, recorded $\int \mathcal{L}=5.08 \mathrm{fb}^{-1}$

- 2012 dataset at $\sqrt{s}=8 \mathrm{TeV}$, recorded $\int \mathcal{L}=21.3 \mathrm{fb}^{-1}$

- presenting selected recent results only, all results on https://twiki.cern.ch/twiki/bin/view/AtlasPublic/BPhysPublicResults 


\section{Associated production of prompt and non-prompt $\mathrm{J} / \psi$ mesons and $Z$ boson}


Associated production of quarkonia with additional objects on LHC:

- single parton scattering (SPS)

- double parton scattering (DPS)

Event selection: $J / \psi \rightarrow \mu^{+} \mu^{-}, Z \rightarrow e^{+} e^{-}$or $\mu^{+} \mu^{-}$

- $Z$ : muons $p_{\mathrm{T}}>24 \mathrm{GeV}$ or electrons $p_{\mathrm{T}}>15 \mathrm{GeV}$, isolation requirement on leptons and trigger match for at least 1 lepton

- $m\left(Z_{\text {cand }}\right)=m\left(Z_{P D G}\right) \pm 10 \mathrm{GeV}$

- $J / \psi: p_{\mathrm{T}}>8.5 \mathrm{GeV},|\eta|<2.1$, leading muon $p_{\mathrm{T}}>4 \mathrm{GeV}$,

- dataset 2012, $20.3 \mathrm{fb}^{-1}$
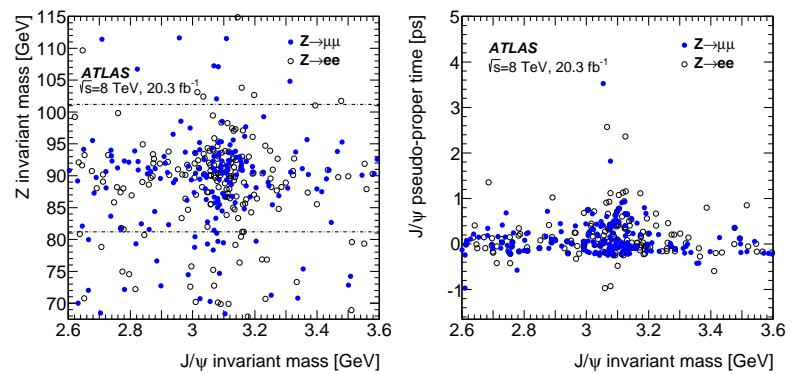


\section{Associated production of $J / \psi$ and $Z$}

Fit $J / \psi$ mass and pseudo-proper time:

Prompt $J / \psi+\mathrm{Z}$ :

- $56 \pm 10 \pm 3$ events

- significance $>5 \sigma$

Non-prompt $J / \psi+\mathrm{Z}$ :

- $95 \pm 12 \pm 8$ events

- significance $>9 \sigma$

Azimutal angle between $\mathrm{J} / \psi$ and $Z$ :

- DPS expecting flat

- SPS peaking at high $\Delta \phi$
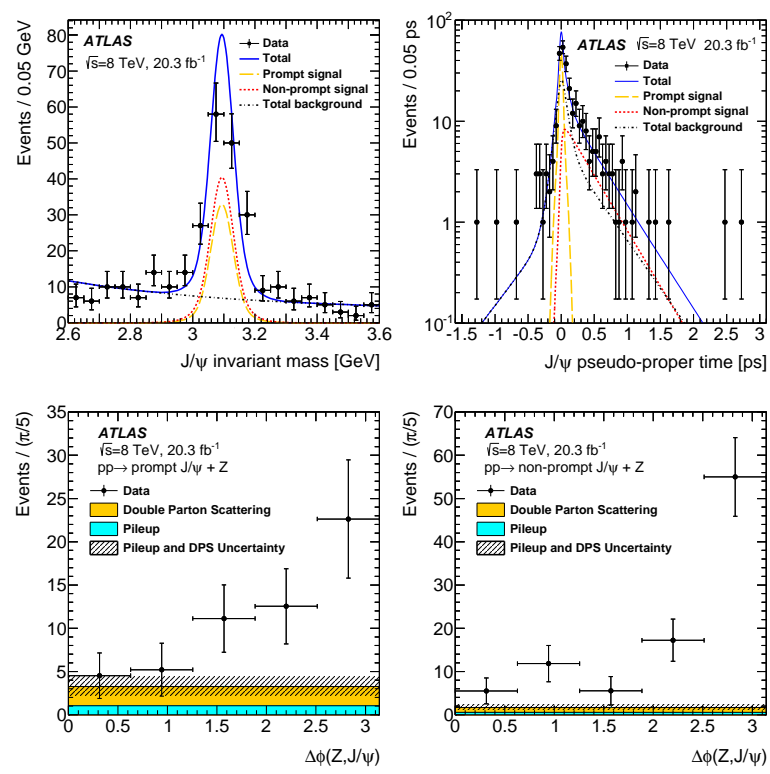


\section{Associated production of $J / \psi$ and $Z$}

- normalised to the inclusive $Z$ cross-section

- fiducial, inclusive (SPS+DPS) and DPS-substracted cross-section

- compared to theoretical predictions (LO colour-singlet mechanism, NLO colour singlet and colour octet)

- models underestimate the SPS contribution
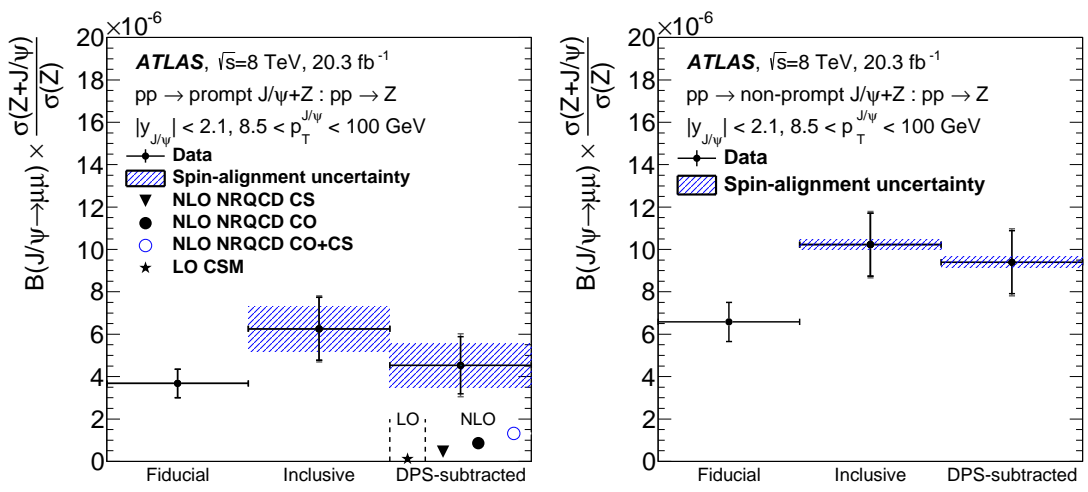


\section{Associated production of $\mathrm{J} / \psi$ and $Z$}

Measured differential production cross-section as a function of $p_{\mathrm{T}}$ (again normalised to the inclusive $Z$ cross-section)
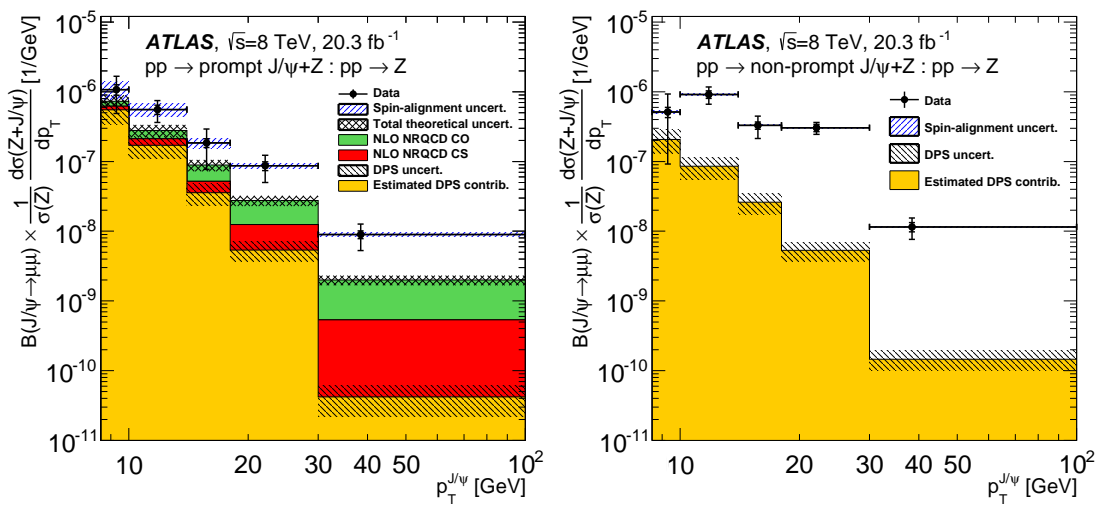


\section{Associated production of $J / \psi$ and $Z$}

- can set upper limit on DPS contribution (assuming independent hard scattering in DPS)

- that gives lower limit on effective cross-section of DPS

$$
\sigma_{\text {eff }}>5.3 \text { (3.7) mb at } 68 \text { (95)\% C.L. }
$$
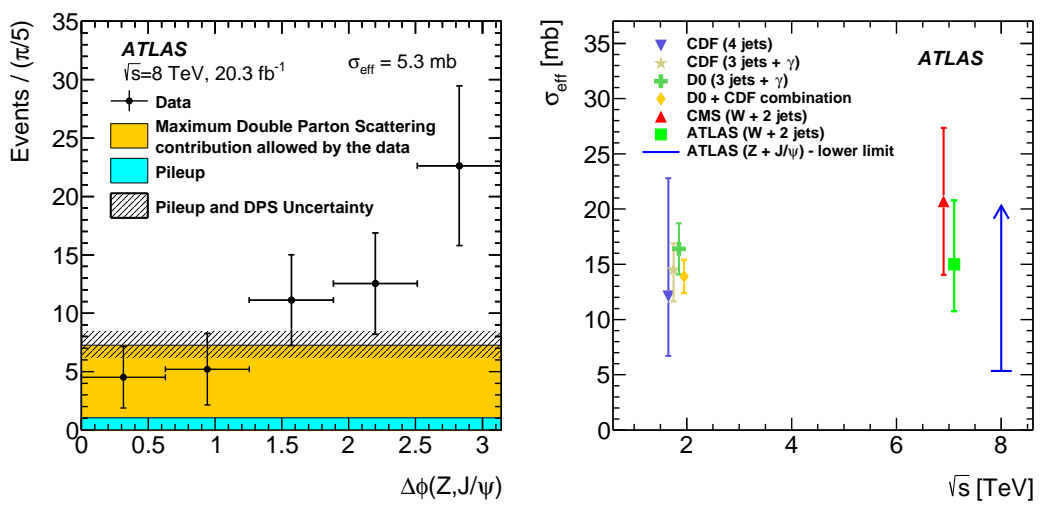


\section{Parity-violating asymmetry parameter $\alpha_{b}$ and helicity amplitudes of $\Lambda_{b}^{0} \rightarrow J / \psi \Lambda^{0}$}




\section{$\Lambda_{b}^{0} \rightarrow J / \psi\left(\mu^{+} \mu^{-}\right) \Lambda^{0}\left(\mathrm{p} \pi^{-}\right)$helicity}

Phys. Rev. D 89 (2014) 092009

Selection:

- $\mathrm{J} / \psi: 2.8 \mathrm{GeV}<\mathrm{m}_{\mu \mu}<3.4 \mathrm{GeV}$

- $\Lambda^{0}: 1.08 \mathrm{GeV}<\mathrm{m}_{\mathrm{hh}}<1.15 \mathrm{GeV}$

- $\Lambda_{b}^{0}: 5.56 \mathrm{GeV}<\mathrm{m}_{\mathrm{J} / \psi^{\sim}}<5.68 \mathrm{GeV}$

- cascade topology: $\chi^{2} / \mathrm{N}_{\text {dof }}<3$,

$\mathrm{L}_{x y}>10 \mathrm{~mm}, \tau_{\Lambda_{b}}>0.35 \mathrm{ps}$

- $1400 \Lambda_{b}^{0}$ and $\bar{\Lambda}_{b}^{0}$ in 2011 dataset Analysis:

- decay described by 4 helicity amplitudes

- parity violating asymmetry parameter $\alpha_{b}=\left|a_{+}\right|^{2}-\left|a_{-}\right|^{2}+\left|b_{+}\right|^{2}-\left|b_{-}\right|^{2}$

- full angular PDF

- used method of moments $F_{i}$

- lifetime and mass measurement in Phys. Rev. D87 (2013) 032002

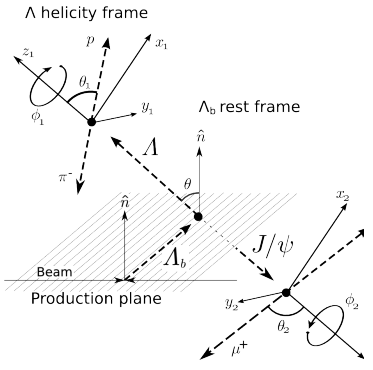

$J / \Psi$ helicity frame

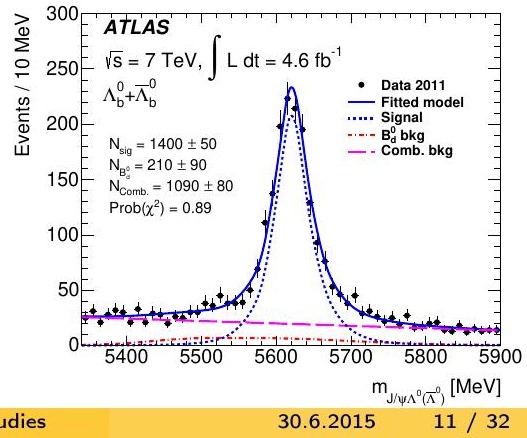




\section{$\Lambda_{b}^{0} \rightarrow J / \psi\left(\mu^{+} \mu^{-}\right) \Lambda^{0}\left(\mathrm{p} \pi^{-}\right)$}

Check fit: compared $F_{i}$ for data with weighted signal+background $\mathrm{MC}$ Results:

$$
\begin{aligned}
& \left|\mathrm{a}_{+}\right|=0.17_{-0.17}^{+0.12} \text { (stat) } \pm 0.09 \text { (syst) } \\
& \left|\mathrm{a}_{-}\right|=0.59_{-0.07}^{+0.06} \text { (stat) } \pm 0.03 \text { (syst) } \\
& \left|\mathrm{b}_{+}\right|=0.79_{-0.05}^{+0.04} \text { (stat) } \pm 0.02 \text { (syst) } \\
& \left|\mathrm{b}_{-}\right|=0.08_{-0.08}^{+0.13} \text { (stat) } \pm 0.06 \text { (syst) } \\
& \alpha_{b}=0.30 \pm 0.16 \text { (stat) } \pm 0.06 \text { (syst) }
\end{aligned}
$$

- $\Lambda^{0}$ and $\mathrm{J} / \psi$ are highly polarized in direction of their momenta

- $\alpha_{b}$ value consistent with $\mathrm{LHCb}$ : $0.05 \pm 0.17$ (stat) \pm 0.07 (syst)

(Phys. Lett.B724 (2013) 27)

- inconsistent with $\mathrm{pQCD}$ $\alpha_{b}=(-0.17,-0.14)$ and HQET $\alpha_{b}=0.78$ at a level of 2.6 and $2.8 \sigma$
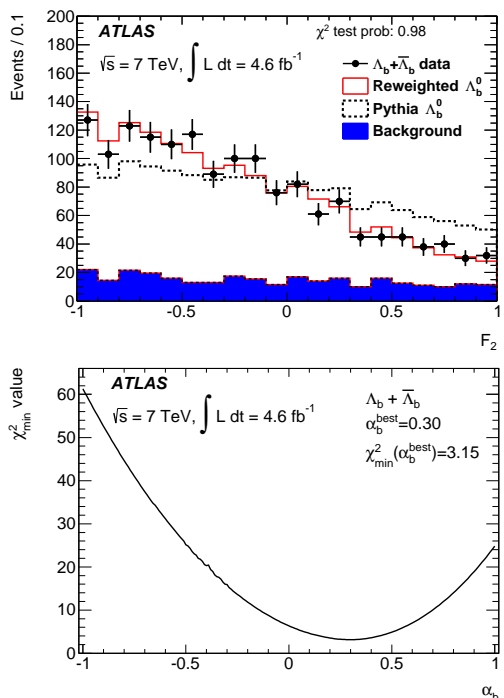


\section{Study of the $B_{c}^{+} \rightarrow J / \psi D_{s}^{+}$and $B_{c}^{+} \rightarrow J / \psi D_{s}^{*+}$ decays}


$B_{c}^{+} \rightarrow J / \psi D_{s}^{+}$and $B_{c}^{+} \rightarrow J / \psi D_{s}^{*+}$ decays ATLAS-CONF-2015-014

$B_{c}^{ \pm}$is an interesting object with two different heavy-flavour quarks

- decay $B_{c}^{ \pm} \rightarrow J / \psi\left(\mu^{+} \mu^{-}\right) \pi^{ \pm}$observed in 2011 data (ATLAS-CONF-2012-028)

- process $\bar{b} \rightarrow \bar{c} c \bar{s}$ allows $B_{c}^{+}$decay to charmonium and $D_{s}^{(*)+}$

- subsequent $D_{s}^{*+} \rightarrow D_{s}^{+}\left(\gamma / \pi^{0}\right)$ and $D_{s}^{+} \rightarrow \phi\left(K^{+} K^{-}\right) \pi^{+}$

- $B_{c}^{+} \rightarrow J / \psi D_{s}^{*+}$ helicity amplitudes: $A_{++}, A_{--}$and $A_{00}$
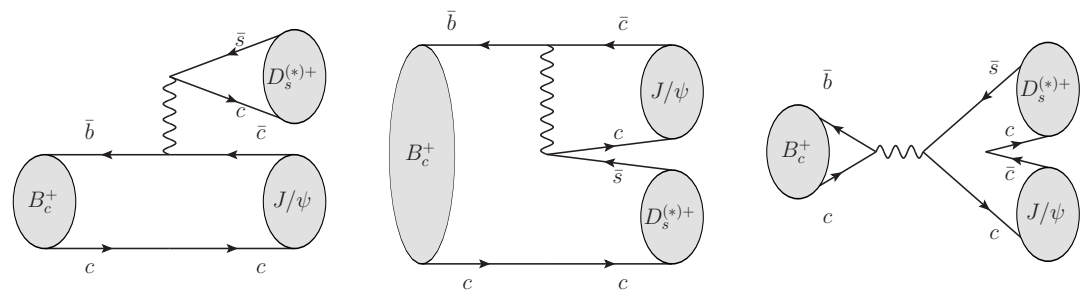


\section{$B_{c}^{+} \rightarrow J / \psi\left(\mu^{+} \mu^{-}\right) D_{s}^{+}\left(K^{+} K^{-} \pi^{+}\right)$event selection}

$\mathrm{J} / \psi$ :

- 2 muons $p_{\mathrm{T}}>3 \mathrm{GeV}$

- $\chi^{2} /$ n.d.f $(J / \psi)<15$

- $m(J / \psi)$ in 2800-3400 MeV

$D_{s}^{+}:$

- 3 tracks fitted to common vertex

- tracks $p_{\mathrm{T}}>1 \mathrm{GeV}$

- $\chi^{2} /$ n.d.f $<8$

- $m\left(K^{+} K^{-}\right)$within $\pm 7 \mathrm{MeV}$ from $m_{P D G}$

- $m\left(K^{+} K^{-} \pi^{+}\right)=1930-2010 \mathrm{MeV}$

- $0.15 \mathrm{~mm}<L_{x y}<10 \mathrm{~mm}$

- pion pointing angle: $\cos \theta^{*}<0.8$

- $\left|\cos ^{3} \theta(K \pi)\right|>0.15$ in c.m.s $(\phi)$
$B_{c}^{+}$: fitting cascade decay

- constraining $J / \psi$ and $D_{s}^{+}$ masses to PDG values

- $\chi^{2} /$ n.d.f $<3$

- $p_{\mathrm{T}}>15 \mathrm{GeV}$

- $d_{0}<0.1 \mathrm{~mm} ; z_{0} \cdot \sin \theta<0.5$ $\mathrm{mm}$

- $0.1 \mathrm{~mm}<L_{x y}<10 \mathrm{~mm}$

- $D_{s}^{+}$pointing: $\cos \theta^{*}>-0.8$

- $1547 \mathrm{~J} / \psi D_{s}^{+}$candidates selected 


\section{$B_{c}^{+} \rightarrow J / \psi D_{s}^{(*)+}$ fit}

2D unbinned maximum likelihood fit of mass and helicity angle $\theta^{\prime}\left(\mu^{+}\right)$:

- $B_{c}^{+} \rightarrow J / \psi D_{s}^{+}$signal

- $B_{c}^{+} \rightarrow J / \psi D_{s}^{*+}$ with $A_{ \pm \pm}$

- $B_{c}^{+} \rightarrow J / \psi D_{s}^{*+}$ with $A_{00}$

- background
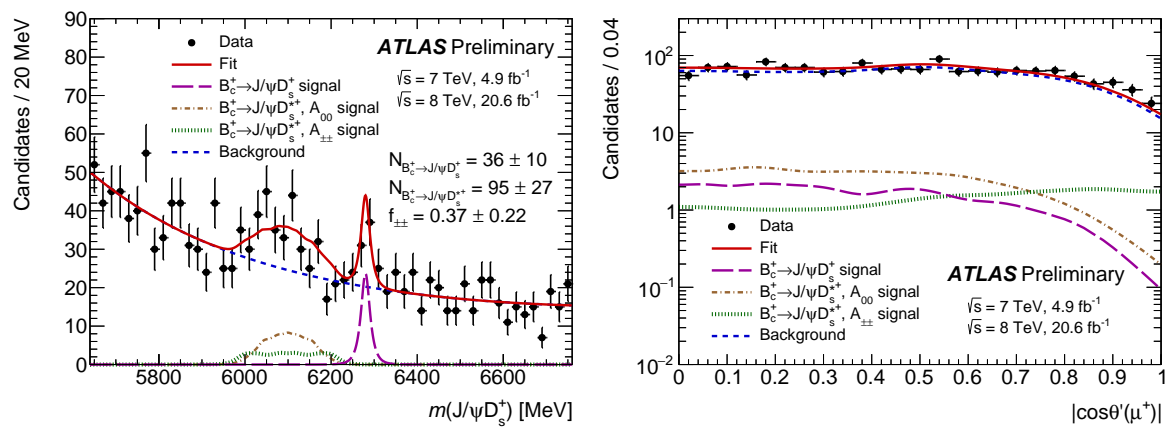

Measured transverse polarisation fraction of $B_{c}^{+} \rightarrow \mathrm{J} / \psi D_{s}^{*+}$

$$
\Gamma_{ \pm \pm} / \Gamma=0.38 \pm 0.23 \text { (stat) }{ }_{-0.07}^{+0.06} \text { (syst) }
$$




\section{$B_{c}^{+} \rightarrow J / \psi D_{s}^{(*)+}$ branching fraction ratios}

Ratios of branching fractions with $B_{c}^{+} \rightarrow \mathrm{J} / \psi \pi^{+}$as reference decay:

$$
\begin{aligned}
\mathcal{R}_{D_{s}^{+} / \pi^{+}} & =\frac{\mathcal{B}\left(B_{c}^{+} \rightarrow J / \psi D_{s}^{+}\right)}{\mathcal{B}\left(B_{c}^{+} \rightarrow J / \psi \pi^{+}\right)}=3.8 \pm 1.1 \text { (stat) }{ }_{-0.6}^{+0.2} \text { (syst) } \pm 0.2\left(\mathcal{B}_{D_{s}}\right) \\
\mathcal{R}_{D_{s}^{*+} / \pi^{+}} & =\frac{\mathcal{B}\left(B_{c}^{+} \rightarrow J / \psi D_{s}^{*+}\right)}{\mathcal{B}\left(B_{c}^{+} \rightarrow J / \psi \pi^{+}\right)}=10.3 \pm 3.1 \text { (stat) }{ }_{-1.5}^{+0.8} \text { (syst) } \pm 0.6\left(\mathcal{B}_{D_{s}}\right)
\end{aligned}
$$

Ratio of $B_{c}^{+} \rightarrow J / \psi D_{s}^{(*)+}$ branching fractions:

$$
\mathcal{R}_{D_{s}^{*+} / D_{s}^{+}}=\frac{\mathcal{B}\left(B_{C}^{+} \rightarrow J / \psi D_{s}^{*+}\right)}{\mathcal{B}\left(B_{C}^{+} \rightarrow J / \psi D_{s}^{+}\right)}=2.7 \begin{aligned}
& +1.1 \\
& -0.8 \text { (stat) }
\end{aligned}
$$

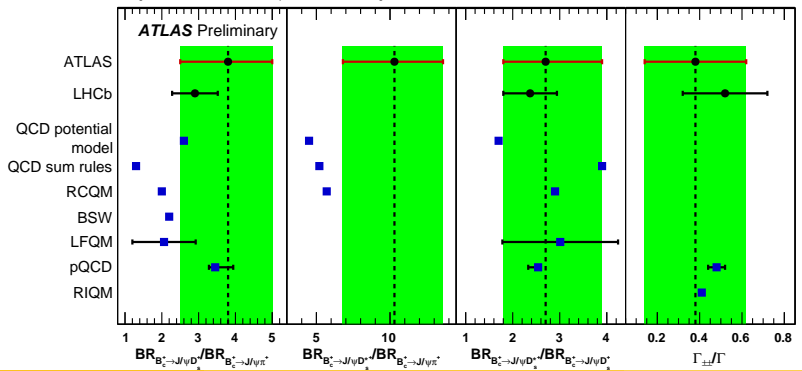




\section{Observation of an excited $B_{c}^{ \pm}$meson state}




\section{$B_{c}^{ \pm}$states}

Excited states of $B_{c}^{ \pm}$are predicted by NRQCD, pQCD and Lattice:

- $1 \mathrm{~S}$ and $2 \mathrm{~S}$ states have a mass splitting for $\mathrm{0}^{+}$and $0^{-}$components

- ATLAS not sensitive enough to distinguish them: missing soft gamma, mass resolution

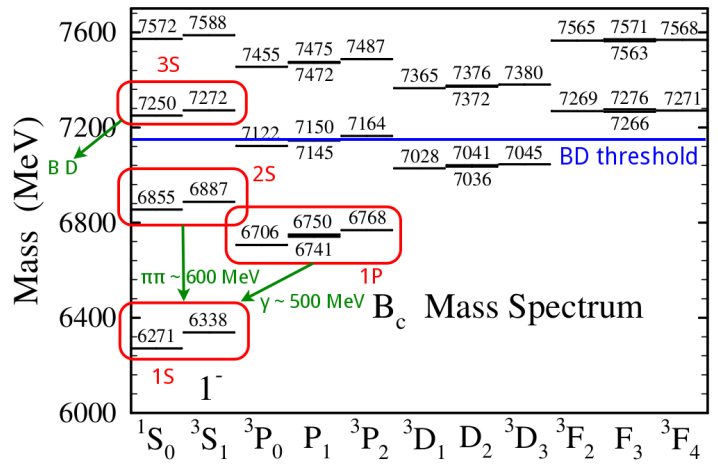

Phys. Rev. D 70 (2004) 054017 


\section{$B_{c}^{ \pm}(1 S) \rightarrow J / \psi\left(\mu^{+} \mu^{-}\right) \pi^{ \pm}$: selection and fit}

$B_{c}^{ \pm}(1 S)$ selection optimised separately for 2011 (2012) dataset:

- $p_{\mathrm{T}}(\mu)>4$ and $6 \mathrm{GeV}$

- $\chi^{2} /$ n.d.f $(J / \psi)<15$

- $m(J / \psi) 3 \sigma$ from nominal mass

- $\chi^{2} /$ n.d.f $\left(B_{c}\right)<2.0(1.5)$

- $p_{\mathrm{T}}\left(B_{c}\right)>15(18) \mathrm{GeV}$

- $d_{x y}^{0} / \sigma\left(d_{x y}^{0}\right)\left(\pi^{+}\right)>5(4.5)$

Unbinned fit of mass distribution:

- Gaussian with per-candidate error

- exponential background
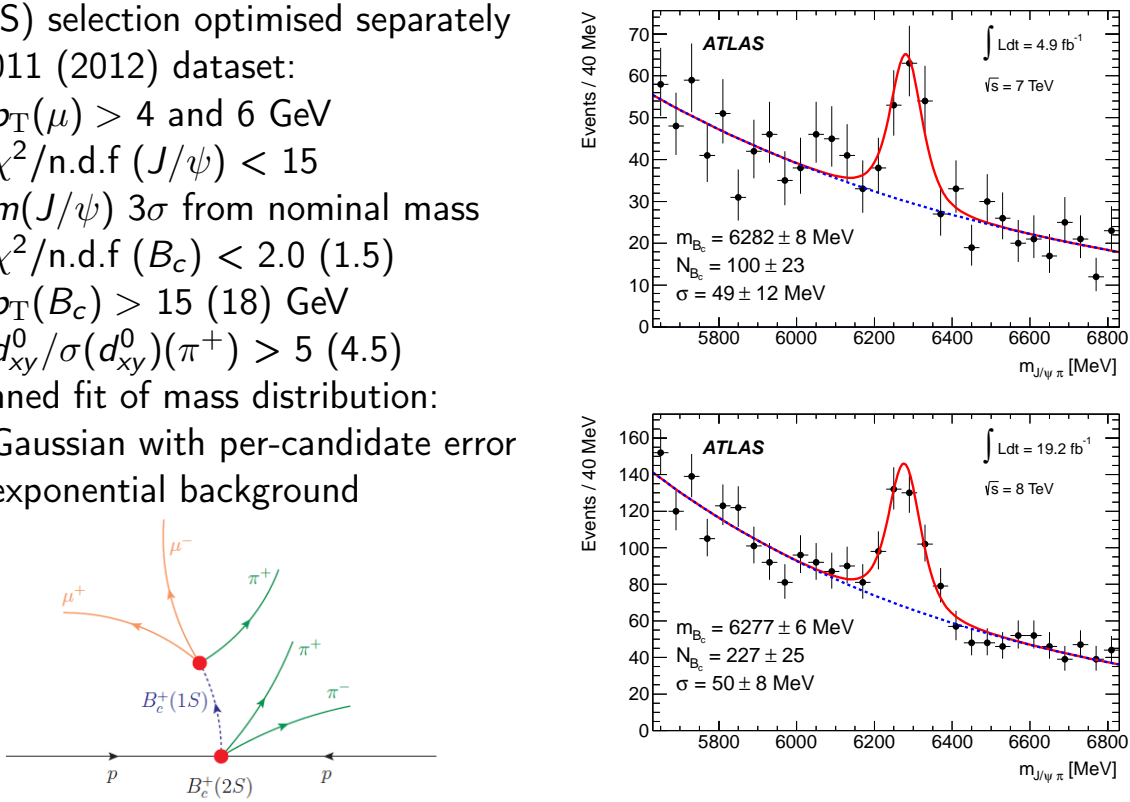


\section{$B_{c}^{ \pm}(2 S) \rightarrow B_{c}^{ \pm}(2 S) \pi^{+} \pi^{-}$}

Candidate selection:

- $B_{c}^{ \pm}(1 \mathrm{~S})$ within $3 \sigma$ from fitted mass

- $p_{\mathrm{T}}\left(\pi^{ \pm}\right)>400 \mathrm{MeV}$

- if several candidates per event, select the one with the best cascade fit $\chi^{2}$

Extended unbinned fit of $Q$-value:

$Q_{B_{c}^{ \pm} \pi \pi}=m\left(B_{c}^{ \pm} \pi \pi\right)-m\left(B_{c}^{ \pm}\right)-2 m(\pi)$

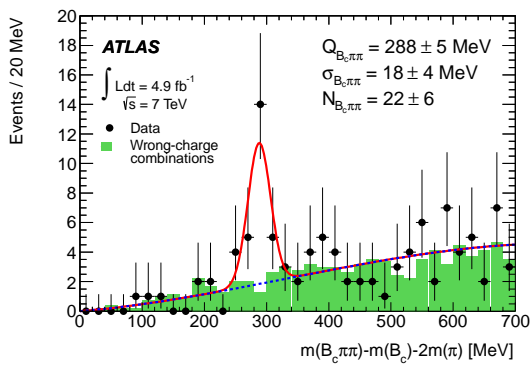

- Gaussian signal

- 3rd order polynomial for background

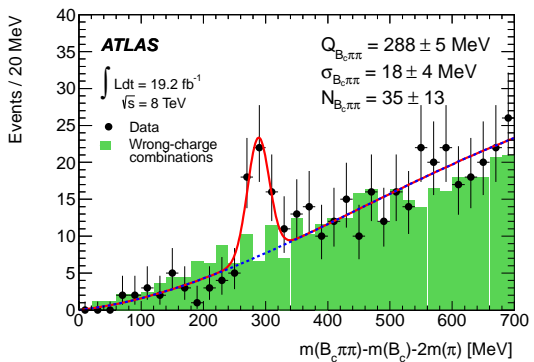




\section{Observation of an excited $B_{c}^{ \pm}$state}

Significance calculated using toy studies to account for "look elsewhere effect"

- $3.7 \sigma$ in $7 \mathrm{TeV}$ data

- $4.5 \sigma$ in $8 \mathrm{TeV}$ data

- combined significance is $5.2 \sigma$

Observed new state at

$Q=288.3 \pm 3.5$ (stat) \pm 4.1 (syst) $\mathrm{MeV}$

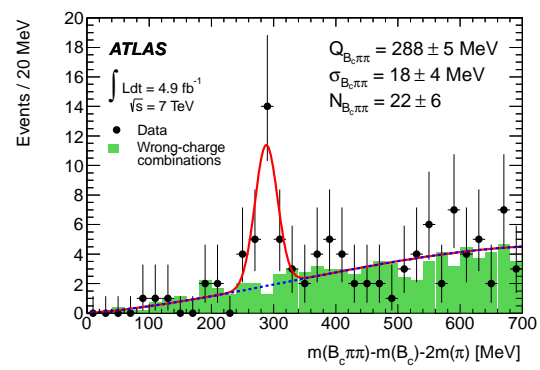

corresponding to a mass

$m=6842 \pm 4$ (stat) \pm 5 (syst) MeV

consistent value of $6835-6917 \mathrm{MeV}$ predicted by various theories

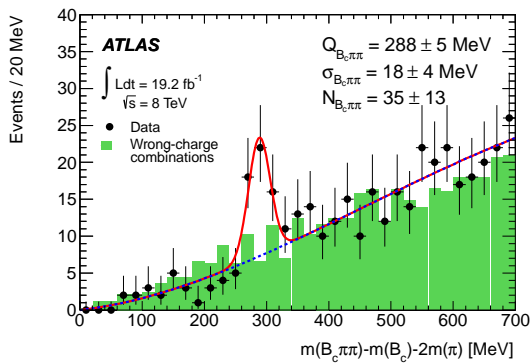




\section{Search for $X_{b}$ and other hidden-beauty states using $\pi^{+} \pi^{-} \Upsilon(1 S)$ channel}




\section{Search for $X_{b}$}

Heavy-quark symmetry suggests existence of $X_{b}$ analogous to $X(3872)$ observed by Belle in $B^{+} \rightarrow K^{+} X(3872)$ with $X(3872) \rightarrow J / \psi \pi^{+} \pi^{-}$ (Phys. Rev. Lett. 91 (2003) 262001)

- search for $X_{b}$ in $\Upsilon(n S) \pi^{+} \pi^{-}$

- mass predictions around $10.5 \mathrm{GeV}$

Data and selection:

- selected by di-muon trigger with $p_{\mathrm{T}}>4 \mathrm{GeV}, m(\mu \mu)=8-12 \mathrm{GeV}$

- $16.2 \mathrm{fb}^{-1}$ at $8 \mathrm{TeV}$

- reconstructed 6 million $\Upsilon(1 S)$ and 0.2 million $\Upsilon(2 S)$ candidates Expected number of $X_{b}$ events

$$
N=N_{2 S} \cdot R \cdot \frac{A}{A_{2 S}} \cdot \frac{\varepsilon}{2 \varepsilon_{2 S}}
$$

where $R \equiv(\sigma \mathcal{B}) /(\sigma \mathcal{B})_{2 S}$ is production rate relative to $\Upsilon(2 S)$ 


\section{Search for $X_{b}$}

- bin data according to $\mathrm{S} / \mathrm{B}$ in $p_{\mathrm{T}}$ and $\cos (\theta)$

- $\Upsilon(1 S) \pi^{+} \pi^{-}$mass distribution for most sensitive bin $-\Upsilon(2 S)$ and $\Upsilon(3 S)$ peaks

- invariant mass fits for $\Upsilon(2 S)$ (barrel, endcap) and $\Upsilon(3 S)$ :
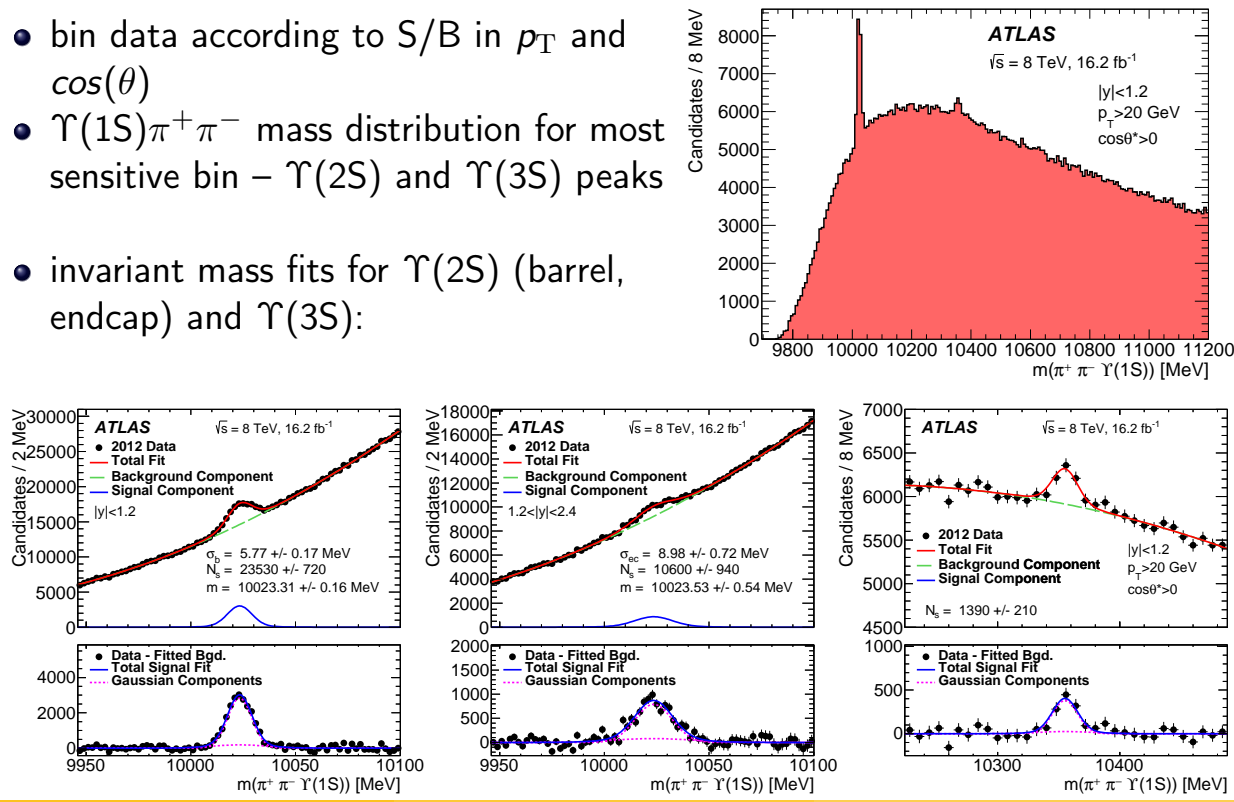

Ina Chalupková (Charles University)

ATLAS heavy flavour studies

30.6.2015

$25 / 32$ 


\section{Search for $X_{b}$}
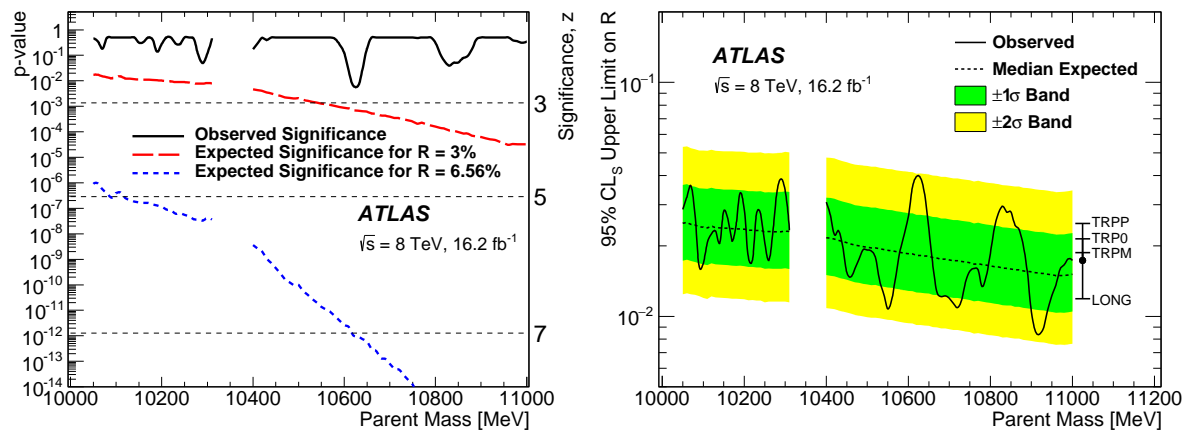

- no evidence for $X_{b}$ found

- considered also different spin alignments for $X_{b}$

- upper limits $0.8-4 \%$ (depending on mass) - currently the best limits

- no evidence for production of $\Upsilon\left(1^{3} D_{J}\right)$ triplet, $\Upsilon(10860)$ or $\Upsilon(11020)$ 


\section{Heavy flavour physics in LHC Run 2}

B-physics programme will follow the Run 1 approach:

- precision measurements of rare processes, focus on potential beyond-SM effects

- heavy flavour production at $13 \mathrm{TeV}$

- searches for new and exotic states and decay modes

Detector upgrades during long shutdowns:

- already installed IBL, additional muon chambers, consolidation

- improved tracking, impact parameter and decay time resolution

- study potential for CPV measurements, e.g. $B_{s}^{0} \rightarrow J / \psi \phi$

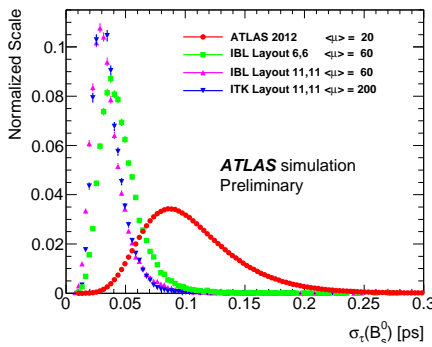

\begin{tabular}{l|c|c|c|c|}
\hline & 2011 & 2012 & \multicolumn{2}{|c|}{$2015-17$} \\
\hline Detector & current & current & \multicolumn{2}{|c|}{ IBL } \\
Average interactions per $\mathrm{BX}<\mu>$ & $6-12$ & 21 & \multicolumn{2}{|c|}{60} \\
Luminosity, $\mathrm{fb}^{-1}$ & 4.9 & 20 & \multicolumn{2}{|c|}{100} \\
\hline Di- $\mu$ trigger $p_{\mathrm{T}}$ thresholds, GeV & $4-4(6)$ & $4-6$ & $6-6$ & $11-11$ \\
Signal events per fb & 400 & 4320 & 3280 & 460 \\
Signal events & 4400 & 86400 & 327900 & 45500 \\
Total events in analysis & 130000 & 550000 & 1874000 & 284000 \\
\hline MC $\sigma\left(\phi_{s}\right)$ (stat.), rad & 0.25 & 0.12 & 0.054 & 0.10 \\
\hline
\end{tabular}




\section{Summary}

The large amount of Heavy Flavour data collected by ATLAS is potentially sensitive to New Physics. Presented analyses:

- associated production of $J / \psi$ and $Z$

- asymmetry parameter and helicity amplitudes of $\Lambda_{b}^{0} \rightarrow J / \psi \Lambda^{0}$

- $B_{c}^{+} \rightarrow J / \psi D_{s}^{*+}$

- $B_{c}^{ \pm}(2 S)$ observation

- search for $X_{b} \rightarrow \Upsilon(n S) \pi^{+} \pi^{-}$

Few more measurements and searches from Run1 dataset to be completed soon and we are collecting first data from Run2.

Thank you for your attention.

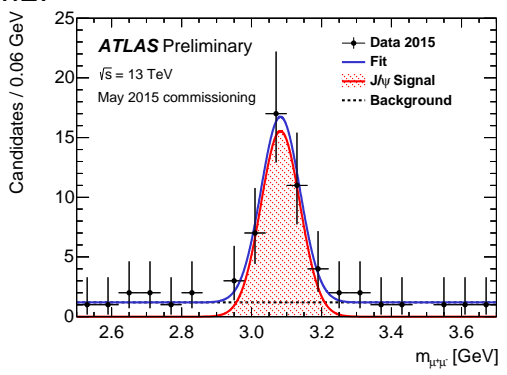




\section{Backup slides}




\section{Associated production of $\mathrm{W}$ and prompt $\mathrm{J} / \psi$

Probes quarkonium production mechanism, sensitive to multiple parton interactions.

Selection:

- prompt $\mathrm{J} / \psi$ via mass and pseudo-proper time

- $\mathrm{W}^{ \pm}$muon trigger, identified by $\mu$ and missing $p_{\mathrm{T}}$

- fit $\mathrm{W}^{ \pm}$transverse mass with multijet background

- 29 events with $\mathrm{W}^{ \pm} \mathrm{J} / \psi$

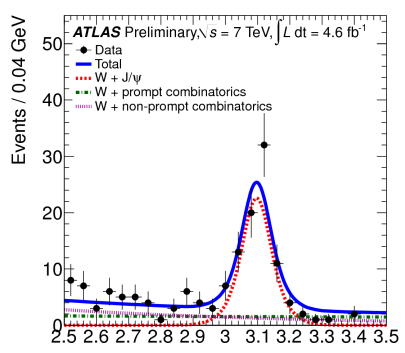

$\mu^{+} \mu^{-}$Invariant mass [GeV]

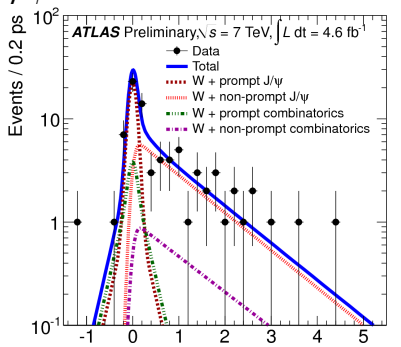

$\mathrm{J} / \psi$ Pseudo-proper time [ps]

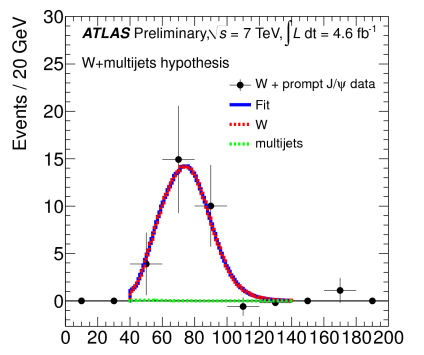

W Transverse Mass [GeV]

$30 / 32$

$-1$




\section{Associated production of $\mathrm{W}$ and prompt $\mathrm{J} / \psi$}

- double parton scattering estimate $\sim 40 \%$ from

$$
\mathrm{d} \sigma_{J / \psi \mid W}=\sigma_{W} \otimes \sigma_{J} / \psi / \sigma_{\text {eff }}
$$

- extract inclusive (SPS+DPS) cross-section ratio

- comparison with theory: measured rate underestimated (but large uncertainties of data)
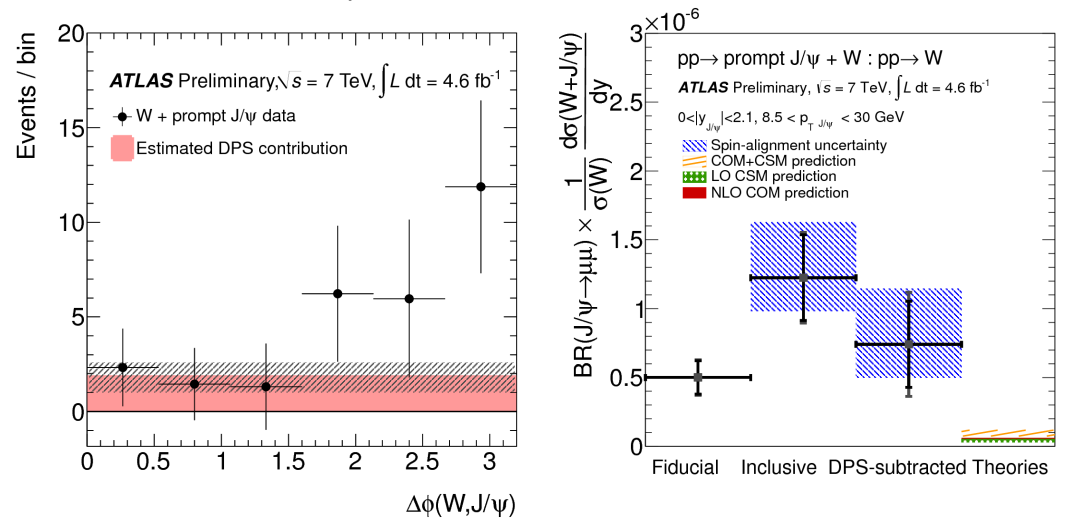


\section{$B_{s}^{0} \rightarrow J / \psi \phi$ analysis}

- tagged analysis using 2011 data $\left(4.9 \mathrm{fb}^{-1}\right)$

- measured

$$
\begin{aligned}
\phi_{s} & =0.12 \pm 0.25 \text { (stat) } \pm 0.05 \text { (syst) rad } \\
\Delta \Gamma_{s} & =0.053 \pm 0.021 \text { (stat) } \pm 0.010 \text { (syst) } \mathrm{ps}^{-1}
\end{aligned}
$$

- about to release analysis using 2012 data

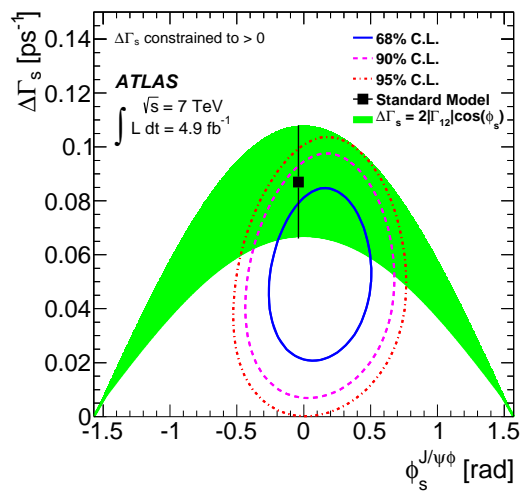

